\title{
Dissection of corticothalamic feedback pathway from V1 to dLGN in mouse
}

\author{
Sayumi Okigawa ${ }^{1}$, Nao Morimoto ${ }^{1,2}$, Fumitaka Osakada ${ }^{1,2,3}$
}

${ }^{1}$ Laboratory of Cellular Pharmacology, Graduate School of Pharmaceutical Sciences, Nagoya University, Japan, ${ }^{2}$ Laboratory of Neural Information Processing, Institute for Advanced Research, Nagoya University, Japan, ${ }^{3}$ PRESTO, Japan Science and Technology Agency, Japan

\section{[background]}

The mammalian brain is a functional system controlled by complex neural networks, composed of various cell types with genetic diversity. Analyzing neural circuits including feedforward and feedback pathways is central to understanding causes of neurological/psychiatric disorders and developing new strategies to treat them.

We focus on feedback pathway in the mouse visual system from the layer 6 (L6) of the primary visual cortex (V1) to the dorsal lateral geniculate nucleus (dLGN) of the thalamus. The thalamus and cortex are interconnected via feedforward and feedback projections. Feedforward pathway has parallel processing streams from dLGN to V1. However, it remains unclear how the feedback pathway organizes and functions, particularly, how different L6 cell types connect to two regions (shell and core) of dLGN.

To understand the roles of the feedback connections, we anatomically analyze them by virus tracing and genome editing.

[methods]

C57BL/6 adult mice received AAV injections into dLGN. Whole brain was harvested and sectioned for histological analysis. Viral vectors for CRISPR/Cas9-mediated Knock-In and trans-synaptic tracing were generated to target particular cell types and identify their connectivity.

[results]

The expression of tdTomato via AAV2-hsyn1-tdTomato injection into dLGN was restricted to the surface/dorsal part of dLGN, or the shell. Co-injection of AAV2-hsyn1-cre with AAV9-CAG-FLEX-TVA-mCheery specifically introduced TVA in the shell. From these results, we performed monosynaptic rabies virus tracing combined with EnvA/TVA and EnvB/TVB systems to visualize individual inputs to either core or shell.

L6 neurons are also heterogeneous in cell morphology and projection pattern. To genetically target them, we screened their specific markers using Allen Brain Atlas RNA-sequencing data and identified three genetically distinct L6 cell types projecting to dLGN. To analyze the projection patterns, morphology and function of each L6 cell type, we took a knocked-in strategy at the marker gene locus using homology-independent-targeted-integration (HITI) devised from CRISPR/Cas9 system.

[conclusions]

We successfully identified at least three distinct L6 cell types connecting to dLGN. Each cell type has its own expression pattern, morphology and neuronal connectivity. Our viral and genetic approaches combined with in vivo imaging will facilitate understanding roles of feedback connections in visual information processing. 